\title{
A Transdisciplinary Conceptual Framework for the Early Identification of Risks for Communication Disorders in Young Children
}

\author{
Alta Kritzinger and Brenda Louw', Louis M Rossetti² \\ 1 Clinic for High Risk Babies \\ Centre for Early Intervention in Communication Pathology \\ Department of Communication Pathology \\ University of Pretoria \\ ${ }^{2}$ Centre for Communicative Disorders \\ University of Wisconsin Oshkosh, USA
}

\begin{abstract}
The early identification of infants with communication disorders or at risk for communication delays is still one of the biggest challenges of early communication intervention (ECI) and threatens to compromise its efficacy. Current approaches to the early identification of young children at risk for communication disorders involve strategies aimed at the identification of general developmental delays and may not be sufficiently sensitive and specific enough to detect the early stages of communication disorders. The risks for mortality and health are often identified early in life, but the concomitant risks for communication disorders in the same young children are frequently not identified at that opportune time. The current study involved a descriptive survey, describing the identification of communication disorders in 153 subjects, whose data was stored in and retrieved from a customized ECI database system. The findings revealed that the subjects were assessed at the average age of 18 months, but that identifications of risk conditions occurred prenatally, at birth, after the perinatal period and later in life. The time of identification related to the different communication disorders identified in the subjects and caregivers played a major role in detecting the first signs of communication disorders in their children. In order to provide a guideline for clinical practice, a transdisciplinary conceptual framework towards a coordinated effort for the early identification of risks for communication disorders in young children is proposed.
\end{abstract}

KEY WORDS: Caregivers, communication disorders, early communication intervention, early identification, transdisciplinary conceptual framework

\section{INTRODUCTION}

The process by which a family with an infant at risk for, or already presenting with a communication disorder, enters an early communication intervention (ECI) programme is by way of identification of the risk condition. The importance of the earliest possible time of identification of a condition disrupting the typical communication development of a young child is now widely recognized and advocated in the field of ECI (Eddey, Robey, Zumoff \& Malik, 1995). According to Rossetti (1993) the early identification of infants eligible for ECI and the extent of parental involvement in the intervention process are the two strongest factors proven to significantly enhance the efficacy of the services. Furthermore, the efficacy of early identification of communication disorders is based on the assumptions that the successful treatment of all communication disorders depends on early detection and treatment of the disorder or risk factors leading to a delay (ASHA, 1991) and that a strong correlation exists between communication skills and future school success (Capute, Palmer \& Shapiro, 1987).
Yet, the early identification of infants with communication disorders or at risk for communication delays is still one of the biggest challenges of ECI and threatens to compromise its efficacy (Bland, 1996; Squires, Nickel \& Eisert, 1996). According to Kochanek and Buka (1995) available epidemiological data reveals exceedingly low identification rates of children with developmental disabilities from birth to three years of age. Alternatively, the number of infants and toddlers with identified disabilities represents a small proportion of those children later identified with disabilities at ten years of age (Kochanek \& Buka, 1995).

Some of the difficulties inherent in the early identification of communication disorders are the nature of identification programmes, the kind of identification strategies to apply to different populations of young children at-risk, the coordination of the different approaches and the implementation of these approaches in different contexts.

Current approaches to the early identification of young children at risk for communication disorders involve unidisciplinary or multidisciplinary strategies aimed at the identification of general developmental 
delays and may not be sufficiently sensitive and specific enough to detect the early stages of communication disorders, such as infants and toddlers with autistic spectrum disorders (Wetherby, Prizant and Hutchinson, 1998). The problem with unidisciplinary or multidisciplinary actions is also that there is limited interaction across the different disciplines and early signs of communication disorders may not be "recognised as such in a young child, or relayed to a speech-language therapist or an audiologist if these professionals are not present at the time of identification of delayed development.

The various identification strategies currently used by speech-language therapists and different professionals involved in the broad field of early intervention include developmental surveillance which entails brief evaluations of developmental skills over a period of time, applied to the total population of children, with the aim to monitor child progress (Kochanek \& Buka, 1995; Squires, et al., 1996); the use of epidemiological data, if available, to provide estimates of the total number of infants in different communities who are at risk for communication disorders (Pattinson \& Hay, 1999; Venter, Christianson, Hutamo, Makhura \& Gericke, 1995); the medical diagnosis of established risk conditions such as chromosomal anomalies or genetic disorders, neurological disorders, congenital malformations, inborn errors in metabolism, sensory disorders, atypical developmental disorders, severe toxic exposure, chronic medical illness and severe infectious disease (Rossetti, 1996) of which the sequelae include communication disorders; strategies to employ caregivers' expert knowledge on their children's development in order to act upon their concerns (Glascoe, 1997) and developmental screening with the sole aim not to exclude children and families from early intervention services (Hock Long, 1996; Kochanek \& Buka, 1995).

Emerging data regarding the efficacy of the various screening strategies for different populations of infants at risk for communication disorders in different settings, indicates that the universal screening of all neonates for hearing loss appears to be a comprehensive strategy, since high risk registers employed in the past fail to identify those infants with a hearing loss of unknown origin (ASHA, 1994; Boswell, 1998; Northern, 1993). Although universal hearing screening is a strategy proposed to improve the early identification rates of infants with hearing loss, it appears not to be a viable identification strategy in South Africa, since no legislation and infrastructure exists to support such a national identification programme.

The use of a risk register as a screening instrument, although found to be ineffective to detect hearing loss of all different origins, may be used in other populations of infants at risk for communication disorders. A checklist - of risk factors known to be associated with delayed communication development and knowledge of specific predictors of communication disorders, such as prolonged feeding problems in infants at biological risk (Kritzinger, 1994) may improve the reliability of screening measures employed. According to Rossetti (1996) knowledge of infant mortality rates and the causes of inf.unt death, provide valuable information about those infants eligible for early communication intervention, since the causes of infant death are also the causes of infant disability. However, the risks for mortality and health are often identified early in life, but the concomitant risks for communication disorders in the same young children are frequently not identified at that opportune time.

Since speech and language disorders have the highest prevalence of any disability in children during the preschool years (Gerber, 1990) and constitute the most common symptom of developmental disability in this population (Rossetti, 1996), it is important to have a coordinated team :approach to identification in early intervention, utilising existing identification strategies for the effective early identification of different types of communication disorders in young children. The purpose of the article is to describe the process of identification of risks for communication disorders in a group of young children in a university-based early communication intervention programme. Based on these findings a transdisciplinary conceptual framework for the early identification risks for communication disorders in young children is proposed which may be applied in different contexts in South Africa.

\section{METHODOLOGY}

The research design was a descriptive survey and the following aims were formulated:

\section{AIMS}

The main aim of the study was to describe the characteristics of a group of young children and their families participating in an university-based ECI programme and explain the initial process of identification of risks for communication disorders in these children, in order to propose a transdisciplinary conceptual framework for an improved identification approach in ECI.

The following sub-aims were formulated:

- To describe when the risks for communication disorders were identified in the children.

- To provide reasons for the course of events:in the identification process and how they pertain to the different risks for communication disorders in the children.

- To indicate who identified the risks for communication disorders in the children.

\section{SUBJECTS}

The subjects employed in the study were 153 infants and toddlers and their families referred to and assessed at CHRIB, an early communication intervention service delivery and student training facility. CHRIB has been operating at the Department of Communication Pathology, University of Pretoria, as a formal assessment and intervention clinic since 1990 (Annual Report 1990, Department of Speech Pathology and Audiology) and now forms part of the Centre for-Early Intervention in Communication Pathology.

The client population of infants and toddlers and their families served by.CHRIB is varied and depends on different referral' sources which includes a Facial 
Deformities Clinic, the Down Syndrome Association, speech-language therapists, other allied health professionals, caregivers themselves and, to a lesser extent, medical professionals.

\section{Sampling and Selection Criteria}

Since no attempt was made to include each subgroup of the population of infants at risk for communication disorders in South Africa in the selection of the subjects, the method of nonprobability convenience sampling was utilized (Leedy, 1997). The subject characteristics in the present study will indicate a bias toward families who can afford to pay for services, as CHRIB is universitybased and not supported by the public health system as in the case of early intervention clinics based at public hospitals and primary health care clinics.

The following two criteria for the selection of the subjects were applied:

- The subjects had to be assessed in CHRIB and had to have undergone the complete CHRIB Assessment Protocol (Louw \& Kritzinger, 1995).
- Each subject had to be assessed by at least three of the team of four qualified and experienced data collectors employed at CHRIB, in order to ensure consistent data collection and recording procedures. (Two data collectors worked simultaneously, the one to interview the parents and the other one to assess the child. The third and fourth data collectors took turns to perform the hearing testing).

By applying the least restrictive subject selection criteria, it could be ensured that the largest possible number of subjects could be recruited within the time constraints of the empirical study. Therefore, all infants and toddlers, together with their families, who were assessed at CHRIB between March 1996 (the starting date of the CHRIB database) and October 1999, and who complied with the selection criteria, were recruited as subjects for the empirical study.

Permission to use the data for research purposes was obtained prior to the assessment. As all families with infants or toddlers referred to and assessed in CHRIB received a letter to request that the data be used for research purposes, all clients were regarded as potential subjects in the empirical study.

\section{Subject Characteristics}

Table 1 Subject characteristics $(\mathrm{N}=153)$

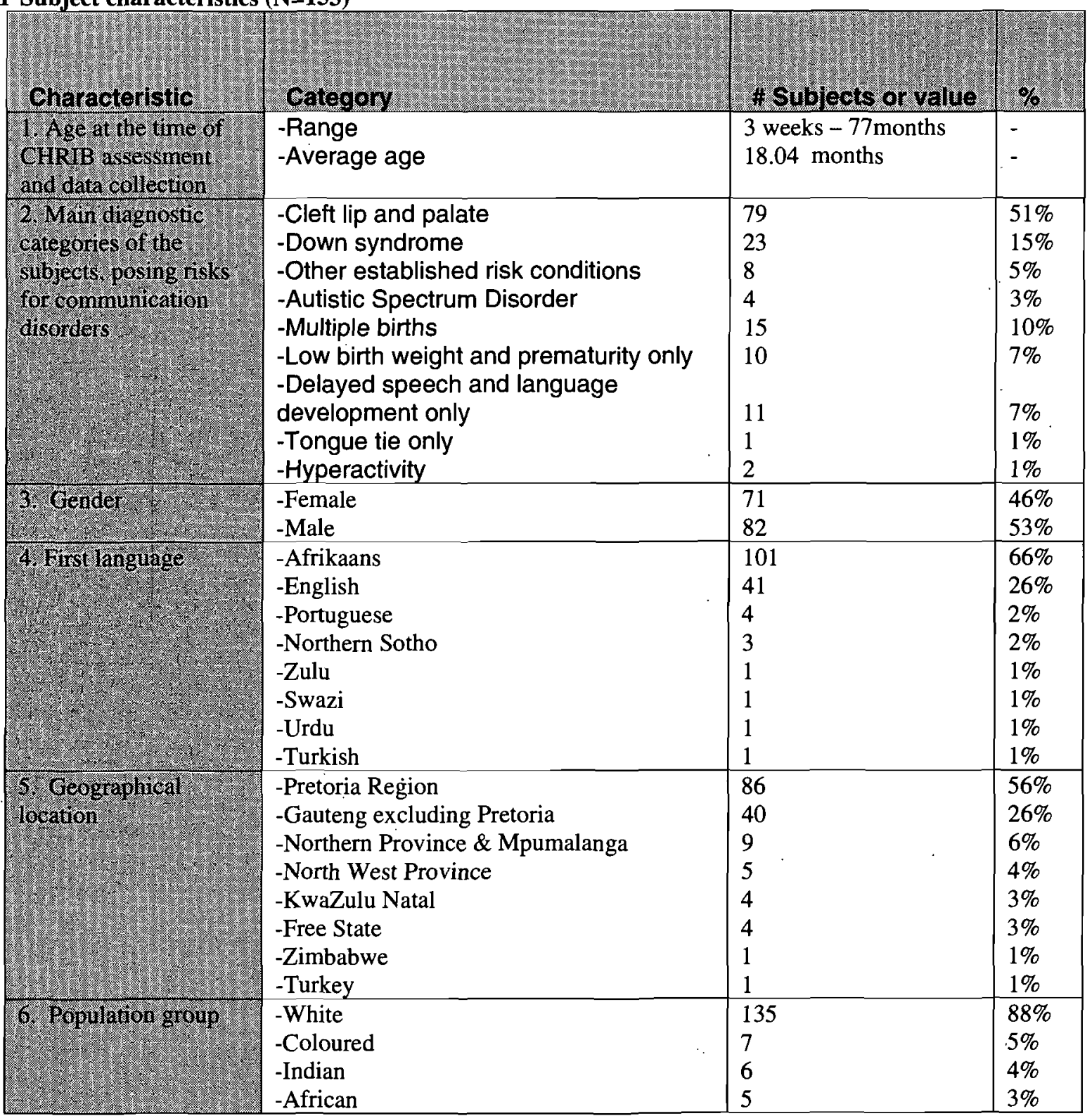


According to Table 1 the main diagnostic categories of the subjects reveal a wide variety of different conditions which pose an established risk for communication disorders, i.e. cleft lip and palate, Down syndrome, autistic spectrum disorders, sensorineural hearing loss, microcephaly, agenesis of the corpus callosum, fetal alcohol syndrome, fetal Roaccutane ${ }^{\circledR}$ syndrome, subarachnoid haemorrhage after surgery to remove a cyst and a suspected genetic disorder in one subject which was unconfirmed at the time of data collection. While established risk conditions occurred in the majority of the subjects, $17 \%$ of the subjects presented with biological risks, such as low birth weight and prematurity, and multiple births. The remaining $9 \%$ of the subjects were categorized according to the main developmental problem they presented with, and could not be classified under the established or biological risk conditions already mentioned. These subjects presented with delayed speech and language development (11 subjects), a tongue-tie (one subject) and hyperactivity (two subjects). Although not clearly evident in Table 1, the different categories, especially the biological risk conditions and speech and language delay, were found not to be mutually exclusive and more than one risk condition occurred in each subject.

The characteristics of the 153 subjects in Table 1 revealed a markedly heterogeneous group of children, representing many of the young children from diverse backgrounds requiring ECI in South Africa. The similarities among the subjects' characteristics disclosed that the majority were boys of about 18 months old, presenting with cleft lip and palate, living in the Pretoria region, are from white families and speak Afrikaans as a home language.

\section{MATERIALS AND APPARATUS}

The materials used in the empirical study were the CHRIB Case History Form (Kritzinger \& Louw, 2000), the CHRIB Assessment Protocol (Louw \& Kritzinger, 1995) and the CHRIB database system, which was modelled on these assessment materials.

The CHRIB Case History Form (Kritzinger \& Louw, 2000 ) is a questionnaire completed by the caregivers prior to the CHRIB assessment and supplies information regarding the child and family's biographical detail, the child's communication problem and diagnosis, the medical and developmental history, the caregivers' concerns about the child's speech-language development and hearing abilities, their efforts to help the child thus far, and their expectations of the assessment.

The nature of the information obtained from the caregivers in the questionnaire were factual data as well as perceptions, providing the researchers with information regarding the caregivers' knowledge and attitudes relating to their child's condition. According to - Girolametto, Weitzman \& Clements-Baartman (1998) and Glascoe (1997), it is now generally accepted that caregivers are reliable sources of information about their children, although they may lack the specialized knowledge to interpret the information correctly. By obtaining the caregivers' opinions about their children's conditions, a family-centered approach to data collection could be achieved.
The CHRIB Assessment Protocol (Louw \& Kritzinger, 1995) was developed to provide the child with opportunities to display a variety of spontaneous and elicited communicative behaviours which were interpreted by utilizing developmental criteria, a variety of developmental scales and a few norm-referenced measurements. The protocol is based on the Stage Process Model of Early Development and adapted from the Holistic Assessment Model for Infants with Cleft Lip and Palate developed by Louw (1986). The protocol covers all developmental areas, adhering to the principle that communication development is closely related to all other aspects of development and an assessment protocol should address these relationships (Louw, 1986; Prizant \& Wetherby, 1995).

The database designed to store the data for the current study is a Microsoft ${ }^{\circledR}$ Access (Aitken, Fulton, Plumley \& Wempen, 1997) relational database management system, structured according to the fifth normal form, and denormalized according to practical needs (Kritzinger, 2000). The database was installed in an Intel Pentium Windows ${ }^{\circledR}$ based system, which contained the necessary hardware specifications in order to support the software of the CHRIB database system.

\section{PROCEDURES}

Following the completion of the CHRIB Case History Form (Kritzinger \& Louw, 2000), the subjects were assessed and all data was recorded and entered into the CHRIB database in the form of text data, numerical data and age interval data. In order to control the accuracy of information in the database, the data entering was done by the first author only.

In order to perform statistical analyses, the data in the CHRIB database system was converted to a flat-file structure in Excel (Microsoft ${ }^{B}$ Office 1997) so that the data could be processed and analyzed by the $S A S \circledR$ (1999) computer package. The different fields or data categories were analyzed by calculating frequency distributions, means, and two-way frequencies in some circumstances. Text data was analyzed and described qualitatively.

\section{VALIDITY AND RELIABILITY}

In order to ensure the validity and reliability of the study, careful consideration was given to the theoretical validity of the materials and the reliability of the assessment instruments, so that the same results will be obtained under comparable conditions. The guidelines for the administration of the different assessment materials were adhered to, in order to ensure consistent data collection procedures by all the data collectors. A statistician was consulted in order to ensure relevant analyses for the particular type of data. The last step employed to demonstrate the internal validity of the empirical study, was to ensure that the final conclusions of the research were adequately supported by the data (De Vos, 1998; Mouton \& Marais, 1990). 


\section{RESULTS AND DISCUSSION}

The advantages of data retrieval by means of an electronic database imply that voluminous amounts of data are readily available and the researcher is intimately involved with the process of data generation and manipulation. Using the CHRIB database system (Kritzinger, 2000) to access the large amounts of data, only the results pertaining to the identification of risks for communication disorders in the subjects were retrieved and will now be discussed.

\section{TIME OF IDENTIFICATION OF RISKS FOR COMMUNICATION DISORDERS IN THE SUBJECTS}

Table 2 Characteristics of the process of identification of risks for communication disorders in the subjects ( $N=153$ )

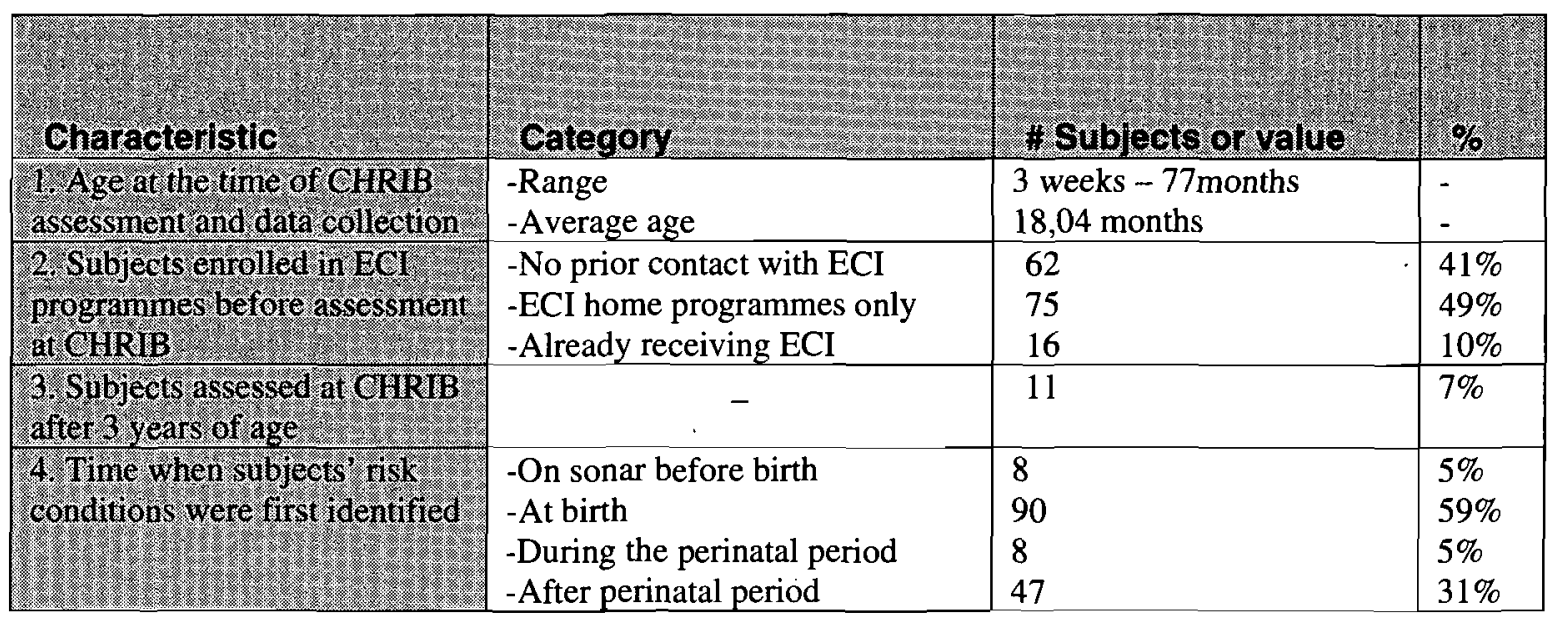

According to Table 2 the subjects presented with a broad spectrum of ages at the time of their CHRIB assessments, from three weeks to 77 months, with an average age of 18 months. The lower margin of the subject ages indicates that CHRIB succeeds in recruiting clients from the earliest possible stage, i.e. the neonatal stage, which is considered as one of the indicators for effective ECI services (Rossetti, 1993). Effectiveness of intervention efforts is also illustrated in the finding that $59 \%$ of the subjects had some form of ECI services before their assessment at CHRIB. On the other hand, the upper limit of 77 months indicates that $11 \%$ of the subjects were only assessed at a very late age.

Table 3 Print out of CHRIB Database Query of subjects older than 3 years ( $N=11$ )

\begin{tabular}{|l|l|l|l|l|l|}
\hline Nr & (D) & Date of Birth & Assessment Date & Diagnosis** & B \\
\hline 1 & 143 & $06 / 10 / 1992$ & $10 / 03 / 1999$ & Q37.4 & Bilateral cleft lip and palate \\
\hline 2 & 120 & $29 / 03 / 1993$ & $05 / 08 / 1998$ & Q35.5.1 & Pierre Robin Sequence \\
\hline 3 & 57 & $06 / 04 / 1993$ & $12 / 03 / 1997$ & F84.01 & Autistic spectrum disorder \\
\hline 4 & 171 & $29 / 07 / 1993$ & $22 / 09 / 1999$ & Q02 & Microcephaly \\
\hline 5 & 136 & $13 / 08 / 1993$ & $10 / 02 / 1999$ & Q37.7.1 & Right-sided cleft lip and palate \\
\hline 6 & 62 & $15 / 02 / 1994$ & $23 / 04 / 1997$ & H91.2 & Sensorineural hearing loss \\
\hline 7 & 78 & $19 / 02 / 1994$ & $06 / 08 / 1997$ & F84.01 & Autistic spectrum disorder \\
\hline 8 & 137 & $27 / 03 / 1995$ & $03 / 02 / 1999$ & Q35.5.1 & Pierre Robin Sequence \\
\hline 9 & 119 & $12 / 04 / 1995$ & $29 / 07 / 1998$ & F80 & Speech and language delay \\
\hline 10 & 157 & $29 / 09 / 1995$ & $07 / 07 / 1999$ & F84.01 & Autistic spectrum disorder \\
\hline 11 & 164 & $29 / 05 / 1996$ & $04 / 08 / 1999$ & Q37.4 & Bilateral cleft lip and palate \\
\hline
\end{tabular}

\section{Key to table headings}

\section{* ID refers to the subject's CHRIB database identification number}

** Diagnostic classifications were made according to the $I C D-10$ (CSS, 1996)

The reasons for the late assessments of the 11 subjects in CHRIB are described in Table 3 involved the following circumstances:

- Two subjects were true late diagnoses and were subsequently diagnosed with established risk conditions. One subject (CHRIB ID 62) was diagnosed with a moderate hearing loss at the assessment and was referred to CHRIB by the caregivers themselves, while the other subject (CHRIB ID/171) was diagnosed with a developmental delay associated with microcephaly and was referred to CHRIB by the Down Syndrome Die Suid-Afrikaanse Tydskrif vir Kommunikasieafwykings, Vol. 48, 2001
Association. As these two subjects had no prior contact with any ECI services and their conditions were identified late, it is of great concern that subjects with conditions which may be readily identified at birth or early in life, went unnoted.

Four subjects (CHRIB ID 57, 78, $119 \& 157$ ) were already receiving speech-language therapy and were referred to CHRIB by their speech-language therapists for consultation with the CHRIB team, as these therapists requested specialist assessment and treatment guidelines. Three of the four subjects were found to present with 
autistic spectrum disorders when assessed at CHRIB and the fourth subject was found to have delayed speech and language development associated with recurrent otitis media.

- The remaining five subjects had various types of cleft lip and palate and were diagnosed as such at birth, but were referred to CHRIB as their speech-language therapists requested a consultation with the CHRIB team, or as a result of the caregivers' late response to an earlier referral to ECI services.

The results indicate that late identifications and referrals occurred very rarely among the 153 subjects and that early identification of risks for communication disorders is happening. The critical importance of time in ECI is, however, emphasized in the example pertaining to the late diagnoses of subjects with autistic spectrum disorders, a concern also described by Squires, et al. (1996) and Wetherby, et al. (1998). According to these authors the diagnoses of autistic spectrum disorders are usually not confirmed until between two and a half and three years of age at best, which indicates that this population of infants who present with a serious communication disorder, is currently underserved by ECI. The late diagnosis of subjects with autistic spectrum disorders assessed in CHRIB corresponds with findings of late diagnosis in clinical practice elsewhere (Squires, et al., 1996; Wetherby, et al., 1998). As the first evident symptoms of autistic spectrum disorders are a delay in speech development, and very little is known about these children's early functioning to accurately diagnose them at a young age (Wetherby, et al., 1998), it appears that ECI can contribute to identify these children earlier.

As further indicated in Table 2 most of the subjects' risk conditions were identified early, i.e., at birth or during the perinatal period, or even prenatally by means of Real Time Ultrasonography in a few instances (See Table 4 as well). The reasons for the successful identification of the subjects at birth or during the perinatal period, may relate to the visual nature of cleft lip and palate and the relative familiarity of health care professionals with Down syndrome (Gerber, 1990; Lubker, 1991). The early identification of subjects indicates that the majority could benefit from ECI at a very early age, if referrals to ECI were successful.

Table 4 Prenatal diagnosis of the subjects' risk conditions ( $N=8$ )

\begin{tabular}{|c|c|c|c|c|}
\hline (1) & Mlont $10 \%$ & Dhagrosis & Oentar & $\begin{array}{l}\text { - Thre of prenabal diagmosis as } \\
\text { reporteg by the caregrers. }\end{array}$ \\
\hline 1 & 19 & Triplets & Male & During pregnancy with sonar \\
\hline 2 & 20 & Triplets & Male & During pregnancy with sonar \\
\hline 3 & 21 & Triplets & Female & During pregnancy with sonar \\
\hline 4 & 55 & Down syndrome & Female & At 2 months of pregnancy \\
\hline 5 & 56 & Cleft lip and palate & Male & Before birth, seen on sonar \\
\hline 6 & 102 & Cleft lip and palate & Male & At 6 months of pregnancy on sonar \\
\hline 7 & 134 & Cleft lip and palate & Female & At 28 weeks of pregnancy on sonar \\
\hline 8 & 169 & Cleft lip and palate & Male & Before birth, seen on sonar \\
\hline
\end{tabular}

Key

\section{Client ID refers to the subject's CHRIB database identification number}

The results presented in Table 4 not only indicate the high technological prenatal care which resulted in the earliest possible diagnosis in some of the subjects, but also reveal a new role for speech-language therapists (Louw \& Kritzinger, 1998), specifically in CHRIB. This role involves the prenatal counseling of families in order to make informed decisions and to be adequately prepared regarding the developmental risks of the diagnosed conditions in their unborn children and the importance of commencing with ECI after birth. The advantage is that caregivers can be prepared prior to the time of active parenting of a child with a risk condition (Louw \& Kritzinger, 1998). The results reported in Table 4 indicate that prenatal informative counselling (Thomson, 1995) and promoting increased public awareness of ECI services should be seen as important functions in CHRIB. Potential caregivers' increased .knowledge of risk conditions for communication disorders can lead to the earlier identification of infants at-risk and eventually to a higher success rate in the prevention of disabilities (Gerber, 1990; Kochanek \& Buka, 1995).

As further indicated in Table 2 and now elaborated in Table 5, the remainder $47(31 \%)$ of the subjects were identified after the perinatal period.
According to Table 5 the identification of risk conditions in $31 \%$ of the subjects after the perinatal period includes a surprisingly wide spectrum of conditions, from established risk conditions such as cleft palate and Down syndrome, which are relatively easily diagnosable at birth, to conditions which only become apparent when the child fails to start talking, to acquired conditions which can only be identified after the causative incident, e.g. acquired sensorineural hearing loss and brain haemorrhage:

Upon closer inspection of the results, the 16 subjects with established risk conditions diagnosed after the perinatal period include five subjects with cleft palate in the absence of a cleft lip, which results in a less visible disorder and possibly easier to overlook at birth, the four subjects with autistic spectrum disorders who were already discussed as a subgroup of children most likely to be identified late, two subjects with Down syndrome and one subject with each of the following disorders: fetal alcohol syndrome, sensorineural, hearing loss, microcephaly, an unknown syndrome which was unconfirmed at the time of data-collection of the study, and agenesis of the corpus callosum. 
REASONS FOR DIFFERENT TIMES OF IDENTIFICATION FOR RISKS FOR COMMUNICAITON DISORDERS IN THE SUBJECTS

Table 5 Number of subjects per risk condition identified after the perinatal period $(N=47)$

\begin{tabular}{|c|c|c|}
\hline 2.:- - & $2.4510 j e c t$ & $.2 \%$ \\
\hline 1. Established risk conditions & 16 & $34 \%$ \\
\hline 2. Acquired risk conditions for communication delay & 2 & $4 \%$ \\
\hline 3. Communication delay associated with low birth weight & 8 & $17 \%$ \\
\hline 4. Communication delay associated with multiple birth & 8 & $17 \%$ \\
\hline 5. Communication delay associated with hyperactivity & 1 & $2 \%$ \\
\hline 6. Hyperactivity only & $I$ & $2 \%$ \\
\hline 7. Communication delay only & 11 & $24 \%$ \\
\hline Total & 47 & $100 \%$ \\
\hline
\end{tabular}

The results relating to the subjects with acquired communication disorders, expressive specific language impairment, autistic spectrum disorders and hyperactivity indicate that not all subjects requiring ECI services could be identified before or at birth, as there were no established or biological markers to indicate a risk status in the subjects. It appears that a conceptual framework for effective ECI services at CHRIB should make provision for earlier identification on a continuum for subgroups of children who can only be identified later in life when their communication development becomes a concern.

The other subjects in Table 5 with conditions identified after the perinatal period, involved the 16 subjects with biological risk conditions present at birth, i.e. low birth weight, prematurity and multiple births. Although presenting with risk conditions early in life, these subjects were only identified when their communication delay became a concern. The two subjects with hyperactivity were both identified by a child psychiatrist, and also only when their communication development became a concern. The one subject with hyperactivity was found to present with a communication delay and the other subject was found to present with normal communication development, but at risk for communication delay when assessed in CHRIB.

The variety of conditions identified in the subjects after the perinatal period provides possible explanations to the concern raised by Kochanek and Buka (1995) that epidemiological data revealed low identification rates of children with disabilities between the ages of birth and three years. Since the problem of late identification in the subjects was not restricted to one type of risk condition only, it could relate to a failure of caregivers and professionals to respond to the first identifiable signs of any of the risk conditions in the subjects. This failure to detect the earliest signs of a communication disorder could relate to a lack of knowledge about the condition as well as the subtlety of its initial stages, and limited transdisciplinary communication across disciplines involved with young children.

Apart from the variety of conditions identified after the perinatal period in the subjects, these subjects were identified at different times in their lives by different people. Each of these variables will be analyzed in Tables 6 and 7 and the ensuing discussions.

\section{Table 6 Ages of subjects identified with communication delay after the perinatal period $(\mathrm{N}=45) *$}

\begin{tabular}{|l|c|c|}
\hline $\begin{array}{l}\text { Subjects identifed ufter the perinatal period } \\
\text { Subjects with 'established risk conditions, } \\
\mathrm{N}=16\end{array}$ & $\begin{array}{c}\text { Average age of } \\
\text { identification of nist } \\
\text { condition }\end{array}$ & $\begin{array}{c}\text { Age of CHRWB } \\
\text { assessment }\end{array}$ \\
$\begin{array}{l}\text { Subjects with communication delay without } \\
\text { established risk conditions, } \mathrm{N}=29\end{array}$ & Average age: $19 \mathrm{~m}$ & Average age: $27 \mathrm{~m}$ \\
\hline
\end{tabular}

Key

* The two subjects, CHRIB ID 89 and 151, with acquired conditions were omitted

According to Table 6 the subjects with communication delay in the absence of established risk factors were identified at the average age of 19 months while the subjects with established risk conditions, even though they were identified after the perinatal period, were identified much earlier, at an average age of 10 months. This finding confirms Rossetti's view (1996) that special attention should be afforded to the identification of infants at-risk, as the identification rate of infants with established risks is much higher.

Further analysis of the subjects in Table 6 clarified the fact that the age of identification of the risk conditions in the subjects did not result in an earlier age of assessment at CHRIB.

Numerous factors, some of which were discussed earlier, can be presented as possible explanations for the time lapse between the age of identification of risk conditions in a subject and the CHRIB assessment. Some of the factors which could have influenced the age of the CHRIB assessment in certain of the subjects relate to late identifications, late referrals, consultations requested by speech-language therapists and audiologists, late response of caregivers to referrals, the unavailability of ECI services in the immediate community, the possibility that the caregivers and/or professionals were not aware of 
ECI and the benefits for very young children, and that a waiting list at CHRIB could have delayed some of the assessments.
Despite the different contributing factors to the late identification of risk conditions in the subjects in Table 6, the subjects' caregivers played an important role in the identification of risk conditions in their young children.

ROLES OF CAREGIVERS AND PROFESSIONALS IN THE IDENTIFICATION OF RISKS FOR COMMUNICATION DISORDERS IN THE SUBJECTS

Table 7 The roles of caregivers and professionals in identifying risk conditions in subjects after the perinatal period $(\mathbf{N}=47)$

\begin{tabular}{|l|l|l|}
\hline \multicolumn{1}{|c|}{ Person who identified risk condition } & \# Subjects & \multicolumn{1}{c|}{$\%$} \\
\hline Caregivers & 30 & $64 \%$ \\
\hline Paediatrician & 6 & $13 \%$ \\
\hline Oral-Facial-Maxillo surgeon & 5 & $11 \%$ \\
\hline Child psychiatrist & 3 & $6 \%$ \\
\hline Play group teacher & 1 & $2 \%$ \\
\hline Neurosurgeon & 1 & $2 \%$ \\
\hline Audiologist & 1 & $2 \%$ \\
\hline Total & $\mathbf{4 7}$ & $\mathbf{1 0 0 \%}$ \\
\hline
\end{tabular}

According to Table 7 most of the subjects, i.e. $64 \%$ were first identified by their caregivers when they noticed a communication delay in their children. The remainder of the subjects, $36 \%$ were identified by professionals as presenting with a condition associated with communication disorder, but it can be reasoned that the caregivers of these subjects had to be concerned about their children in the first instance in order to consult a professional. This confirms the major role that caregivers play in identifying risk conditions in their children and reiterates recent findings that caregivers are correct $79 \%$ of the time when they suspect a problem with their child's development (Glascoe, 1997). It appears, however, that the caregivers' potential to contribute to the early identification and treatment of communication disorders in CHRIB is largely untapped, as they were aware of their children's problems long before they were assessed.

As the effectiveness of ECI depends on the earliest possible identification of those children requiring the services (Rossetti, 1996), the identification of clients needs to be viewed on a continuum. Depending on the particular subgroup of children requiring ECI and the nature of the child's communication disorder, early identification is an ongoing process over time and an ECI service provider must be knowledgeable when certain subgroups of children can be identified and who the likely people are to identify them first. It appears that an early identification model relying on risk factors only will overlook some important subgroups of children requiring ECI. A relevant model has to afford a key position to caregivers as it appears that they are uniquely positioned to identify communication delays and disorders in their children, provided they have the necessary knowledge about ECI. The importance of caregivers at each stage on the continuum of early identification of risk conditions in their children echoes the centrality already afforded to caregivers in the entire service delivery process of ECI (Guralnick, 1997; Rossetti, 1996).

\section{CONCLUSIONS AND IMPLICATIONS}

The results of the study clearly indicated that there were limitations in the early identification of young children at risk for communication disorders. Although most of the established risk conditions for communication disorders in the subjects were well known and commonly occurring congenital disorders, some of these subjects were not identified at the earliest possible times. The implications are that intervention could not start early enough to prevent the sequelae of the condition and that the benefits of early intervention eluded the child and the family.

Rare congenital disorders, such as fetal Roaccutane ${ }^{\circledR}$ syndrome (Briggs, Freeman \& Jaffe, 1994), agenesis of the corpus callosum (Kurtz, Dowrick, Levy \& Batshaw, 1997) and an unconfirmed genetic disorder also presented in the subjects which implies that not all established risk conditions are easily identifiable at birth or later in life. The results further indicated that autistic spectrum disorders and communication delays in the absence of other risk factors, are also conditions which are difficult to diagnose early, most probably as a result of the subtlety of the initial stages of the communication disorder (Wetherby, et al., 1998).

Importantly, the results also indicated subjects with biological risk conditions for communication disorders such as low birth weight and prematurity, multiple births and hyperactivity were identified early in life, but the risk for communication disorders inherent to their conditions, was disregarded. Since early identification and early commencement with intervention is one of the most important predictors of effective ECI services (Rossetti, 1993), the results of the empirical study emphasized the difficulties experienced in clinical practice to attain the goal of the earliest possible identification of all infants with risks for communication disorders. 


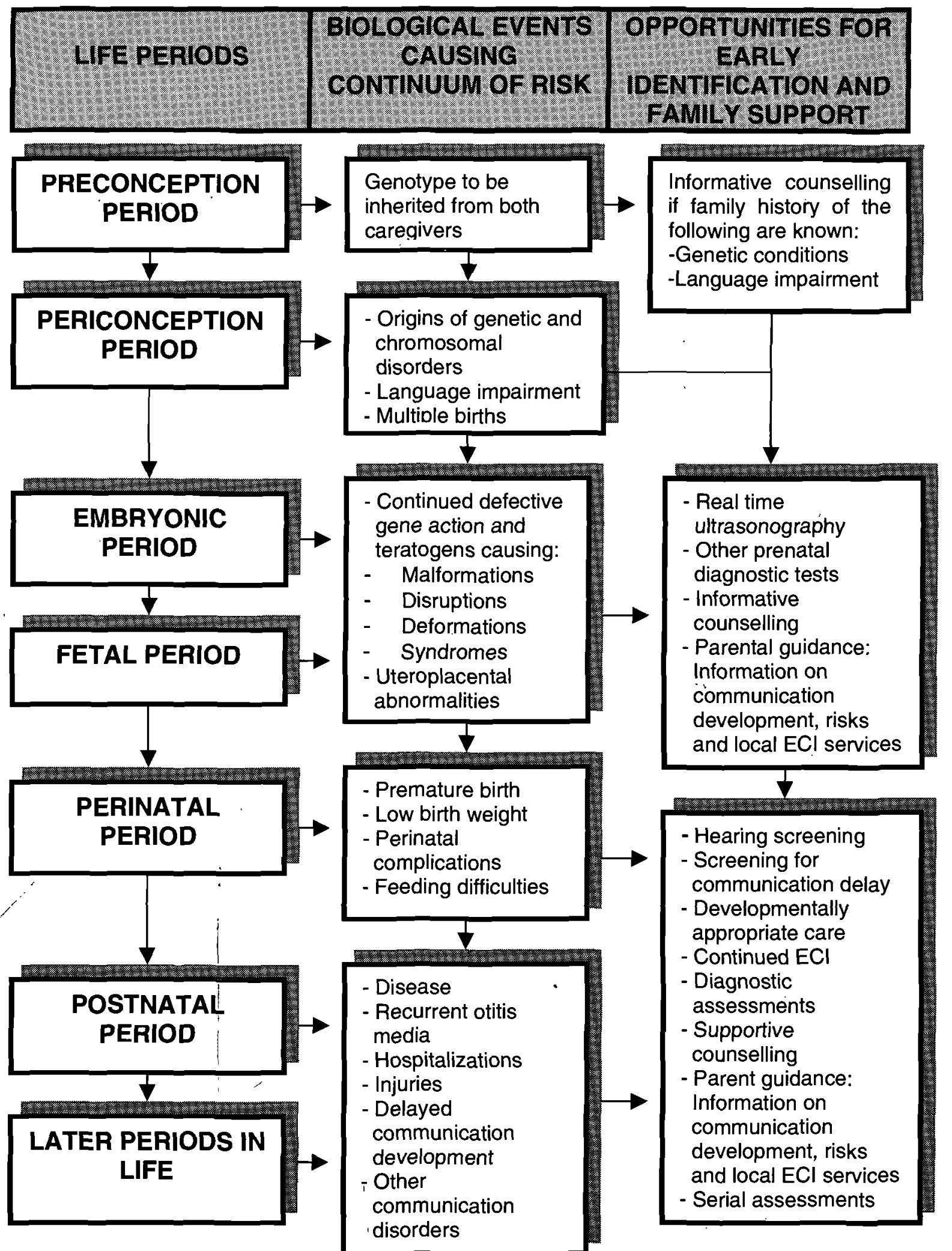

Figure I Transdisciplinary conceptual framework for the early identification of risks for communication disorders Figure I was conceptualized from: Allen, 1995; Glascoe, 1995; Louw \& Kritzinger, 1998; Rossetti, 1996; Rubin, 1995; Sadler, 1995; 1999; Shprintzen, 1997; Thomson, 1995; Tomblin, 1989. 
Since the results identified caregivers as key persons in the identification of risk conditions in their children, the implication is that they need to be equipped with knowledge of the risks for communication disorders in conditions such as low birth weight, prematurity and multiple births occurring in the infants, and how to act on their concerns about the infant's development.

Suggestions for the improvement of early identification in the subjects were indicated in the results. Although caregivers are ideally placed to identify risks for communication disorders in infants as a result of their continuous involvement in their children (Squires, et al., 1996), professionals, assisted by many different technologically based diagnostic procedures, are also present at important times when identification of risk conditions are possible. Different health care professionals are involved at different times in young children's lives, e.g. at the prenatal diagnosis of risk conditions, at birth, during the perinatal period, at primary health care facilities and at family health care practices. Various professionals, equipped with the necessary knowledge about risks for communication disorders and utilizing different identification strategies, are therefore indispensable members of the extended collaborative ECI team responsible for early identification of risks for communication disorders.

Since ECI has the unique opportunity to intervene at the earliest possible time in an infant's life, early identification of risk conditions is the primary function of the ECI approach. Late identification of infants with risks for communication disorders results in late diagnoses, late referrals and late intervention efforts, which compromise the whole process of efficacy of ECI. In order to face the challenge of the multifaceted problem of early identification of communication disorders in young children a transdisciplinary conceptual framework, which addresses the questions of who must be identified, when and by whom, is proposed in Figure I (Kritzinger, 2000).

As illustrated in Figure I the transdisciplinary conceptual framework for early identification of risks for communication disorders is proposed as a guideline for best practice to assist the ECI service provider to identify the critical times of cascading biological events which can negatively impact on the young child's communication development and to identify these events as possible opportunities on a continuum for the identification of risks for communication development in the young child concerned.

Technological advances now allow the prenatal diagnosis of certain risk conditions in children which also affords the ECI service provider the opportunity to provide informative counselling and support to caregivers. The conceptual framework also demonstrates the continuum of risk throughout a young child's life and indicates the different professional functions of $\mathrm{ECI}$ during these periods. The different functions, executed by different professionals involved in the early identification process, include the following:

- Informative and supportive counselling to prospective caregivers, raising public awareness about risks for communication disorders and ECI during the prenatal period. Clinical efforts directed at transdisciplinary work with radiologists, geneticists and nurses involved in the prenatal identification and risk conditions for communication disorders must be explored
(Louw \& Kritzinger, 1998). This way the process of role transition, starting with role extension, and continuing with the phases of role enrichment, expansion, exchange, release and support, of transdisciplinary team interaction can be achieved (Briggs, 1997).

Hearing screening, screening for communication delay, assessment, intervention in the form of developmentally appropriate care and information to caregivers about risks for communication disorders during the perinatal period. Transdisciplinary activities and/or direct involvement by ECI professionals must be initiated during this period where collaboration with paediatricians, nurses in labour wards, well baby nurseries and special care nurseries can improve the age of identification of infants at risk for communication disorders.

Developmental surveillance and serial assessments, followed by treatment by means of parent training, continued parent support and guidance during the postnatal period and later in the child's life. The set of professionals involved in the postnatal health care of infants, such as paediatricians, primary care doctors and nurses, in urban settings or community-based primary health care, as well as those providing daycare to infants, must also be viewed as members of a transdisciplinary team for the early identification of communication disorders in infants.

Lastly, health care professionals, such as primary care doctors and nurses, various medical specialists involved in health care of young children with disabilities, trauma and injuries, and persons involved in day care of young children must be equipped to identify risks for communication disorders later in a child's life and refer the families to local ECI services. The focus of the identification efforts, as role exchange actions in the transdisciplinary process, should also be on communication disorders, such as stuttering, articulation disorders, voice disorders and other communication disorders not discussed in the present study.

The conceptual framework for early identification of risk conditions for communication delay is proposed as a guideline for the ECI service provider to be an active participant, directly or through transdisciplinary action, throughout the critical periods of possible harmful biological and other events in a young child's life'. The aim of the framework is therefore to assist the ECI service provider in identifying key periods where the identification of risk conditions is possible and to develop essential steps to address caregivers' information needs thereby establishing a coordinated effort for early identification of risks for communication disorders. This can only be achieved when holistic partnerships with caregivers and professionals from different disciplines are formed (Briggs, 1997; Rossetti, 1996).

The early identification of children with risks for communication disorders will increase the efficacy of ECI -services and the transdisciplinary conceptual framework for early identification as illustrated in Figure I is proposed to provide a guideline for coordinated action in clinical practice. 


\section{REFERENCES}

Aitken, P., Fulton, J.; Plumly, S. \& Wempen, F. (1997). Microsoft Office ${ }^{\circledR} 97$ Professional 6 in 1. Indianapolis: Que.

Allen, M. C. (1995). Developmental Implications of Intrauterine Growth Retardation. In Blackman, J. A. (Ed.) Medical Aspects of Early Intervention. Gaithersburg: Aspen Publishers, Inc.

ASHA. (1991). The Prevention of Communication Disorders ASHA Supplement Nr 6, 33(9): 15-39.

ASHA. (1994). Joint Committee on Infant Hearing. Position Statement. ASHA, (Dec): 38-41.

Bland, L. E. (1996). Identifying Communication Disorders in Children. Kentucky Medical Journal, 94 (May): 197-201.

Boswell, S. (1998). Newborn Hearing Screening Bill gains support in Congress. ASHA Leader, 3(8), April: $1-2$.

Briggs, M. H. (1997). Building Early Intervention Teams. Gaithersburg: Aspen Publishers, Inc.

Briggs, G. G., Freeman, R. K. \& Yaffe, S. J. (1994). Drugs in Pregnancy and Lactation. Fourth Edition. Baltimore: Williams \& Wilkins.

Capute, A. J., Palmer, F. B. \& Shapiro, B. K. (1987). Using Language to Track Development. Patient Care, November: 60-71.

Central Statistical Services. (1996). ICD-10. International Statistical Classification of Diseases and Related Health Problems. Manual. Pretoria: Central Statistical Services.

De Vos, A. S. (1998). Research at Grass Roots. A Primer for the Caring Professions. Pretoria: J. L. van Schaik Academic.

Eddey, G. E., Robey, K. L., Zumoff, P. \& Malik, Z. Q. (1995). Multidisciplinary Screening in an Arena Setting for Developmental Delay in Children from Birth to Six Years of Age. Infant-Toddler Intervention. The Transdisciplinary Journal, 5(3): 233-242.

Gerber, S. E. (1990). Prevention. The Etiology of Communicative Disorders in Children. New Jersey: Prentice Hall.

Giralometto, L., Weitzman, E. \& ClementsBaartman, J. (1998). Vocabulary Intervention for Children with Down Syndrome: Parent Training using Focused Stimulation. Infant-Toddler Intervention. The Transdisciplinary Journal, 8(2): 109-125.

Glascoe, F. P. (1997). Parents' Concerns about Children's Development: Prescreening Technique or Screening Test? Pediatrics, 99(4): 522-528.

Guralnick, M. J. (1997). The Effectiveness of Early Intervention. Baltimore: Paul. H. Brookes Publishing Company.

Hock Long, L. (1996). Guidelines for Referral. In Kurtz, L. A., Dowrick, P. W., Levy, S. E. \& Batshaw, M. L. (Eds.) Handbook of Developmental Disabilities. Gaithersburg: Aspen Publishers, Inc.

Kochanek, T. T. \& Buka, S. L. (1995). Using Biologic and Ecologic Factors to Identify Vulnerable Infants and Toddlers. In Blackman, J. A. (Ed.) Identification and Assessment in Early Intervention. Gaithersburg: Aspen Publishers, Inc.
Kritzinger, A. M. (1994). Vroeë Kommunikasieontwikkeling van Biologiese Risikobabas. Unpublished M Log Thesis, University of Pretoria.

Kritzinger, A. M. (2000). Establishing a ComputerBased Data System for Early Communication Intervention in South Africa. Unpublished D Phil Thesis, University of Pretoria.

Kritzinger, A. \& Louw, B. (2000). A Comprehensive Assessment Protocol for Infants and Toddlers At Rișk for Communication Disorders. Clinica. Applications in Clinical Practice of Communication Pathology. Monograph 5: 1-18.

Kurtz, L. A., Dowrick, P. W., Levy. S. E. \& Batshaw, M. L. (1996). Handbook of Developmental Disabilities. Gaithersburg: Aspen Publishers, Inc.

Leedy, P. D. (1997). Practical Research. Planning and Design. Sixth Edition. New Jersey: Prentice-Hall, Inc.

Louw, B. (1986). Swart Babas met Gesplete Verhemelte: 'n. Morfofunksionele Studie. Unpublished D Phil Dissertation, University of Pretoria.

Louw, B. \& Kritzinger, A. (1995). CHRIB Assessment Protocol. Department of Communication Pathology, University of Pretoria.

Louw, B. \& Kritzinger, A. (1998). Genetically Based Communication Disorders: Technological Advances Relevant to the Speech-Language Therapist and Audiologist. In Clinica: Applications in Clinical Practice of Communication Pathology. Monograph 3. University of Pretoria.

Lubker, B. B. (1991). Practical uses of Epidemiology in Programs for Infants and Toddlers. Infant-Toddler Intervention: The Transdisciplinary Journal, 1(1): 47 59.

Mouton, J. \& Marais, H. C. (1990). Basic Concepts in the Methodology of the Social Sciences. Pretoria: Human Sciences Research Council.

Northern, J. L. (1993). Infant Hearing Screening. Unpublished lecture delivered at the University of the Witwatersrand, Johannesburg.

Pattinson, R. C. \& Hay, I. T. (1999). A New Educational Strategy Towards Improving Maternal and Neonatal Care. Block 9. Pregnancy, Childbirth, the Neonate, Growth and Development. MB ChB Third Year Curriculum. University of Pretoria.

Prizant, B. M. \& Wetherby, A. M. (1995). Communication and Language Assessment for Young Children. In Blackman, J. A. (Ed.) Identification and Assessment in Early Intervention. Gaithersburg: Aspen Publishers, Inc.

Rossetti, L. M. (1993). Enhancing Early Intervention Services to Infants/Toddlers and their Families. Journal of Childhood Communication Disorders, 15(1): 1-6.

Rossetti, L. M. (1996). Communication Intervention. Birth to Three. San Diego: Singular Publishing Group, Inc.

Rubin, I. L. (1995). Etiology of Developmental Disabilities. In Blackman, J. A. (Ed.) Medical Aspects of Early Intervention. Gaithersburg: Aspen Publishers, Inc.

Sadler, T. W. (1995). Langman's Medical Embriology. Seventh Edition. Baltimore: Williams \& Wilkins.

SAS® Version 8. (1999). Cary, North Carolina: SAS Institute, Inc. 
Shprintzen, R. J. (1997). Genetics, Syndromes and Communication Disorders. San Diego: Singular Publishing Group, Inc.

Squires, J., Nickel, R. E. \& Eisert, D. (1996). Early Detection of Developmental Problems: Strategies for Monitoring Young Children in the Practice Setting. Developmental and Behavioural Pediatrics, 17(6): 420-427.

Thomson, E. J. (1995). A Genetics Primer for Early Service Providers. In Blackman, J. A. (Ed.) Medical Aspects of Early Intervention. Gaithersburg: Aspen Publishers, Inc.

Tomblin, B. J. (1989). Familial Concentration of Developmental Language Impairment. Journal of Speech and Hearing Disorders, 54: 287-295.

Venter, P. A., Christianson, A. L., Hutamo, C. M., Makhura, M. P. \& Gericke, G. S. (1995). Congenital Anomalies in Rural Black South African Neonates. South African Medical Journal, 85: 15-20.

Wetherby, A. M., Prizant, B. M. \& Hutchinson, T. A. (1998). Communicative, Social/Affective, and Symbolic Profiles of Young Children with Autism and Pervasive Developmental Disorders. American Journal of Speech-Language Pathology, 7(2): 79-91.
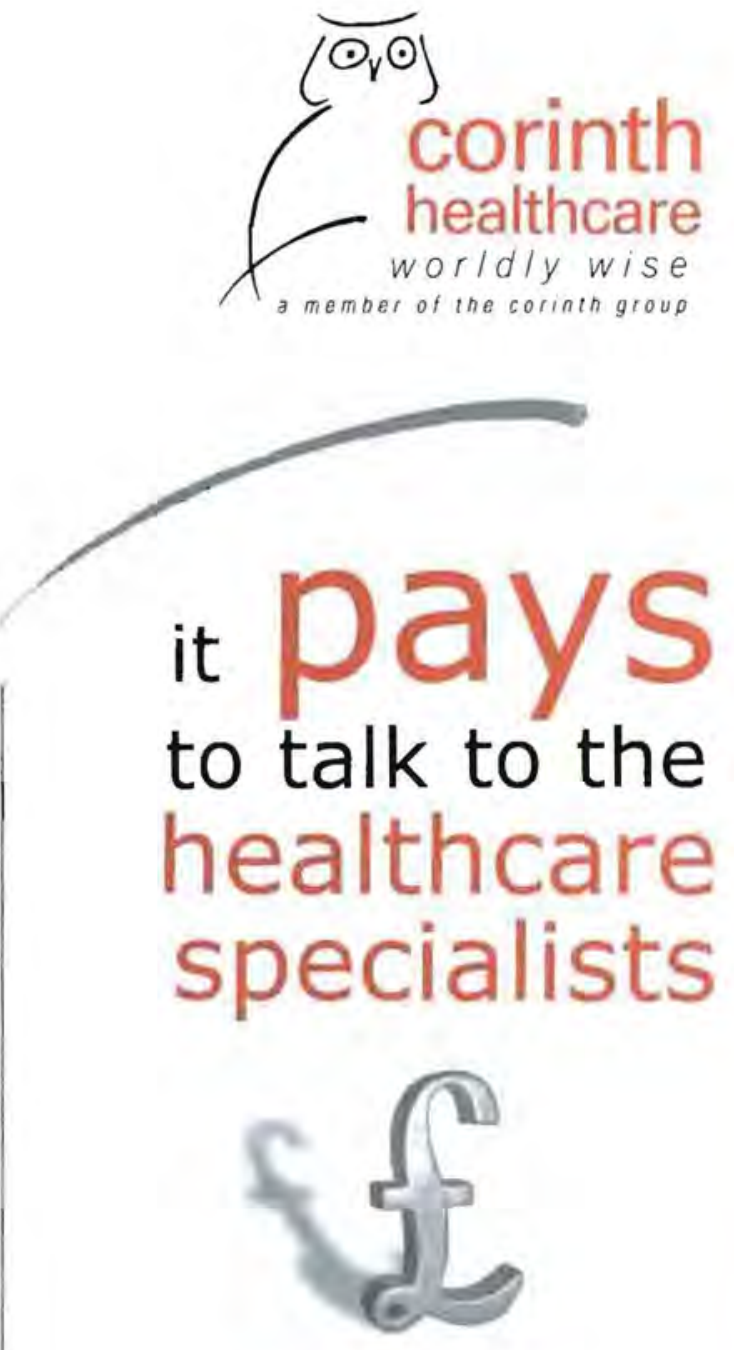

call Corinth, your fast track to the UK 0800201120

E-mail corinth@icon.co.za or corinthdrs@icon.co.za

\section{Speech and Language Therapists}

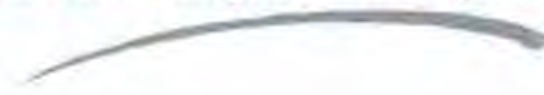

Audiologists Biomedical 5cientists Cardlogriphers Csoicardiac

Technicians Dental Norses

Dieficlans Doctors/Specialists

Medicol Adrain and Clerical

Medical Laboratory Technicians

Medical Secretaries

Occupational Therapists

Phlebotomists Psychiatrists

Physiotherapasts Radiographers

Social Worluers

Speech and Language Therapists

Theatre Nurses 